\title{
Salvage Carbon-ion Radiotherapy for Isolated Lymph Node Recurrence Following Curative Resection of Esophageal Cancer
}

\author{
YUKA ISOZAKI $^{1}$, SHIGEO YASUDA ${ }^{1}$, YASUNORI AKUTSU ${ }^{2}$, HIDEAKI SHIMADA ${ }^{3}$, SHINICHI OKAZUMI ${ }^{4}$, \\ YOSHIHIRO NABEYA ${ }^{5}$, ISAMU HOSHINO ${ }^{5}$, KENTARO MURAKAMI ${ }^{2}$, DANIEL K. EBNER ${ }^{1,6}$, \\ HIROSHI TSUJI ${ }^{1}$, TADASHI KAMADA ${ }^{1}$, SHIGERU YAMADA ${ }^{1}$ and HISAHIRO MATSUBARA ${ }^{2}$ \\ ${ }^{1}$ Hospital of the National Institute of Radiological Science, \\ National Institutes for Quantum and Radiological Science and Technology, Chiba, Japan; \\ ${ }^{2}$ Frontier Surgery Department, Graduate School of Medicine, Chiba University, Chiba, Japan; \\ ${ }^{3}$ Department of Gastroenterological Surgery and Clinical Oncology, \\ Toho University Graduate School of Medicine, Tokyo, Japan; \\ ${ }^{4}$ Department of Surgery, Toho University Sakura Medical Center, Chiba, Japan; \\ ${ }^{5}$ Division of Gastroenterological Surgery, Chiba Cancer Center, Chiba, Japan; \\ ${ }^{6}$ Brown University Alpert Medical School, Providence, RI, U.S.A.
}

\begin{abstract}
Aim: Evaluation of the therapeutic efficacy of carbon-ion radiation therapy for isolated lymph node recurrence following curative resection of esophageal cancer. Materials and Methods: Ten cases with lymph node recurrence after esophageal cancer surgery were treated with carbon-ion radiation therapy. A total of $48.0 \mathrm{~Gy}$ [relative biologic effectiveness (RBE)] was delivered over 3 weeks with a daily dose of 4.0 Gy (RBE). Results: The median follow-up duration was 27.1 months (range=3-92.0 months) after carbon-ion radiation therapy. The local control rates at 2, 3 and 5 years were $92.4 \%$. The overall survival rates at 2, 3 and 5 years were $70.0 \%, 58.3 \%$ and $21.9 \%$. The median survival period was 45.3 months after carbon-ion radiation therapy. There were no toxicities of grade 3 or higher. Conclusion: Carbon-ion radiation therapy may be a safe and effective treatment option for isolated lymph node recurrence after radical surgery for esophageal cancer.
\end{abstract}

Esophageal cancer is a particularly malignant form of digestive disease, and the eighth most common cancer worldwide (1). Esophagectomy remains the standard treatment for patients with resectable disease, but is complicated by a

Correspondence to: Yuka Isozaki, MD, Ph.D., Hospital of the National Institute of Radiological Science, National Institutes of Quantum and Radiological Science and Technology, 4-9-1, Anagawa, Inage Ward, Chiba, 263-0024, Japan. Tel: +81 432063360, Fax: +81 432063345, e-mail: isozakiyu@ chiba-u.jp

Key Words: Esophageal cancer, lymph node recurrence, carbon-ion radiotherapy. high rate of postoperative recurrence and poor survival, even when preliminary investigation suggests that surgery will be curative. According to previous reports, $27-52 \%$ of patients who undergo esophagectomy in the setting of esophageal cancer will go on to develop recurrence (2-9).

It was revealed that lymphadenectomy for lymph node recurrence following curative resection achieved locoregional disease control and offered a favorable outcome, especially for cervical node recurrence $(10,11)$. Several articles showed the clinical usefulness of chemoradiotherapy (CRT) for patients with isolated lymph node recurrence $(2,11)$.

In 1994, clinical studies of carbon-ion radiotherapy (CIRT) began at the National Institute of Radiological Sciences (NIRS), Japan (12). Carbon-ion beams have a high relative biological effectiveness (RBE) with high linearenergy transfer compared to proton or photon beams (13). They are also superior to photon beams in terms of dose distribution and minimization of dose to surrounding normal tissues (13).

The first phase I/II clinical trial for short-course preoperative CIRT for resectable esophageal carcinoma was a dose escalation study carried out between 2004 and 2008 $(14,15)$. Our results suggested that CIRT could be both a highly effective and less toxic therapy (14). A second-phase I/II clinical dose-escalation study of preoperative shortcourse irradiation combined with concurrent chemotherapy was started from 2012 and is currently ongoing $(14,15)$.

To further contribute improvement to the prognosis of esophageal cancer, CIRT for isolated lymph node recurrence after curative resection of esophageal cancer was conducted at a single institution. We retrospectively evaluated the effectiveness and safety of this treatment. 
Table I. Patient clinical characteristics are shown. Four patients had already received radiotherapy for irradiation field.

\begin{tabular}{|c|c|c|c|c|c|c|c|c|c|c|c|c|}
\hline Case & $\begin{array}{c}\text { Age } \\
\text { (years) }\end{array}$ & Gender & r Location & Pathology & $\mathrm{pT}$ & $\mathrm{pN}$ & $\begin{array}{l}\text { Adjuvant } \\
\text { therapy }\end{array}$ & Resection & $\begin{array}{c}\text { DFI } \\
\text { (months) }\end{array}$ & $\begin{array}{l}\text { Site of } \\
\text { recurrence }\end{array}$ & $\begin{array}{c}\text { Tumor } \\
\text { size }(\mathrm{cm})\end{array}$ & $\begin{array}{l}\text { Radiation } \\
\text { field }\end{array}$ \\
\hline 1 & 64 & M & $\mathrm{Lt}$ & SCC & 3 & 3 & NACRT & Esophagectomy & 23.3 & Abdominal & 1.1 & Local \\
\hline 2 & 65 & M & Mt & SCC & 2 & 3 & NACRT & Esophagectomy & 14.9 & Lt recurrent nerve & 3.5 & Local \\
\hline 3 & 67 & M & $\mathrm{Ce}$ & SCC & 4 & 2 & NAC & Esophagectomy & 9.1 & Thoracic & 2 & Regional \\
\hline 4 & 60 & $\mathrm{~F}$ & Ut & SCC & $1 b$ & 2 & NAC & Esophagectomy & 19.7 & Lt supraclavicular & 1.5 & Regional \\
\hline 5 & 66 & $\mathrm{~F}$ & Mt & SCC & 3 & 0 & NACRT & Esophagectomy & 33.6 & Thoracic & 2.5 & Local \\
\hline 6 & 61 & M & Mt & SCC & $\mathrm{U}$ & $\mathrm{U}$ & $\mathrm{AC}$ & Esophagectomy & 19.2 & Lt recurrent nerve & 1.6 & Regional \\
\hline 7 & 71 & M & Mt & SCC & $1 \mathrm{~b}$ & 1 & NAC & Esophagectomy & 15.1 & Rt recurrent nerve & 3.5 & Regional \\
\hline 8 & 60 & M & Mt & SCC & $\mathrm{U}$ & $\mathrm{U}$ & -- & Esophagectomy & 21 & Upper mediastinal & 4.6 & Regional \\
\hline 9 & 68 & M & Mt & SCC & 3 & 3 & NAC & Esophagectomy & 17.3 & Upper mediastinal & 8 & Regional \\
\hline 10 & 72 & $\mathrm{~F}$ & Mt & SCC & $1 \mathrm{~b}$ & 0 & $\begin{array}{l}\text { Prophylactic } \\
\text { radiation }\end{array}$ & $\begin{array}{l}\text { Endoscopic } \\
\text { resection }\end{array}$ & 84.5 & Rt recurrent nerve & 1.8 & Local \\
\hline
\end{tabular}

Ce: Cervical, Ut: upper thoracic, Mt: middle thoracic, Lt: lower thoracic, SCC: squamous cell carcinoma, NACRT: neoadjuvant chemoradiotherapy, NAC: neoadjuvant chemotherapy, AC: adjuvant chemotherapy, DFI: disease-free interval from resection to lymph node recurrence, Lt: left, Rt: right, U: unknown.

\section{Materials and Methods}

Patient eligibility. From March 2000 to August 2016, 10 cases of isolated lymph node recurrence after curative resection for esophageal cancer received CIRT. Eligibility criteria included: i) Confirmed heterochronic isolated lymph node recurrence after curative resection for esophageal cancer, ii) no findings of other metastasis, iii) an expected prognosis of more than 6 months, iv) performance status (PS) 0-2, v) no other serious complications. Exclusion criteria included: i) patients with another primary malignancy, ii) an infection at the tumor site.

Isolated lymph node recurrence was defined as recurrence of a resected primary esophageal carcinoma in one or multiple lymph nodes within a single en-bloc region, within one irradiation field, with no other metastasis at time of presentation. Disease was diagnosed and evaluated with contrast-enhanced computed tomography (CT) scans, positron-emission tomography (PET), ultrasonography and physical findings.

Informed consent was obtained from all patients and the present study was approved by the Institutional Review Board of the NIRS (9404, 9905, 0801G).

Carbon-ion radiotherapy. The carbon ion beam irradiation system has previously been described (16). To reproduce exact body position at the time of treatment, patients were positioned in customized cradles and immobilized with a low-temperature thermoplastic sheet. Using CT images, three-dimensional treatment planning was performed using the Xio software program (ELEKTA, Stockholm, Sweden, and Mitsubishi Electric, Tokyo, Japan), which was developed specifically for CIRT treatment planning.

CIRT was performed once per day, 4 days per week. A total dose of $48.0 \mathrm{~Gy}$ (RBE) was delivered to the planning target volume, with a daily dose of $4.0 \mathrm{~Gy}$ (RBE). The target volume was verified by contrast-enhanced CT and PET. For the first six cases treated, the initial target volume was defined as the lymph node recurrence and the nodal region (including the bilateral superclavicular, mediastinal or abdominal regions). After a total dose of $36 \mathrm{~Gy}$ (RBE), the field was limited to only macroscopic lesions with an added margin of
$1.0 \mathrm{~cm}$ (Figure 1A). For the remaining four patients, who were previously irradiated, local fields with a margin of $1.0 \mathrm{~cm}$ around the macroscopic tumor were used (Figure 1B).

The irradiation dose was calculated for the target volume and surrounding normal tissue and was expressed in Gy (RBE), defined as the carbon physical dose (Gy) multiplied by the RBE value of 3.0 for carbon-ion beams $(12,17)$.

Treatment results and evaluation of adverse events. After treatment, patients were followed-up every 1-3 months for 2 years and then every 3-6 months thereafter using CT and PET scans. Changes in the tumor diameter before and after treatment were evaluated in accordance with the Response Evaluation Criteria in Solid Tumors: Revised guidelines (RECIST version 1.1) (18). The first site of failure was evaluated in terms of local (recurrent lymph node) recurrence, regional field (regional lymph nodes) failure, and distant failure.

The local control, survival, and relapse-free survival rates were calculated using the Kaplan-Meier method. Acute toxicity within 3 months was classified according to the National Cancer Institute Common Toxicity Criteria Version 4.0 (19). Late toxicity was evaluated using the Radiation Therapy Oncology Group/European Organization for Research and Treatment of Cancer scoring system (20).

\section{Results}

Clinical characteristics of patients. Patient clinical characteristics are shown in Table I. The median age was 65 (range $=60-72$ ) years, with seven males and three females. The disease-free interval between curative resection and diagnosis of recurrence ranged from 9.1 to 84.5 months. The lymph node recurrences were within the supraclavicular field in one patient, mediastinal field in eight patients, and abdominal field in one patient. Four patients had already received radiotherapy to the irradiation field. The median maximal coronal diameter of the tumor was $2.3 \mathrm{~cm}$ (range $=1.1-8.0 \mathrm{~cm}$ ). Figure 1 shows 


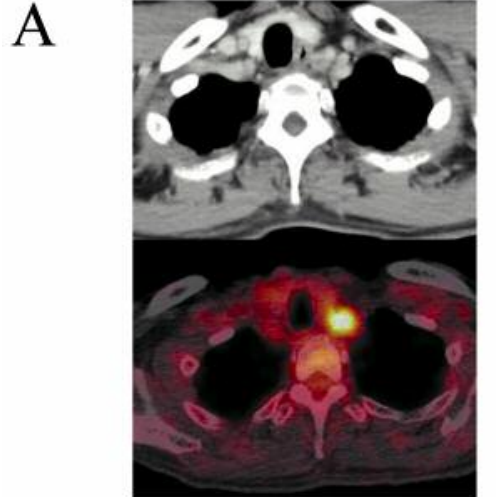

Before CIRT

B

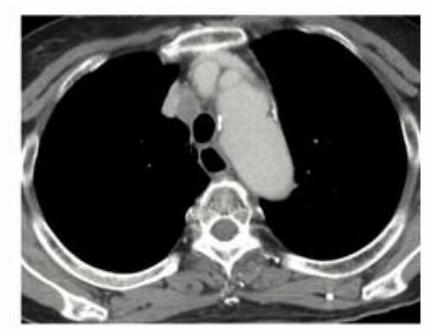

Before CIRT

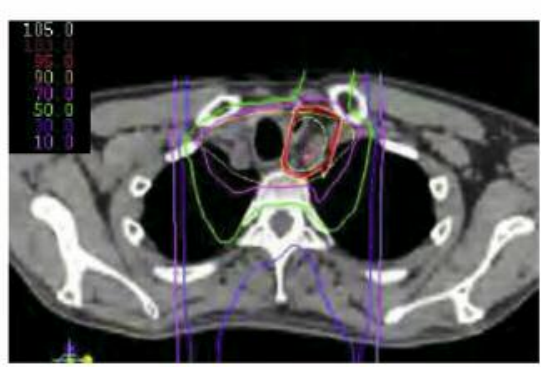

Carbon ion radiation [ 48.0 Gy $(\mathrm{RBE}) / 12 \mathrm{fr}$ ]

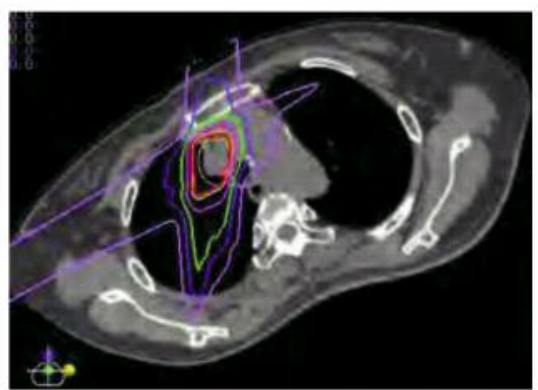

Carbon ion radiation

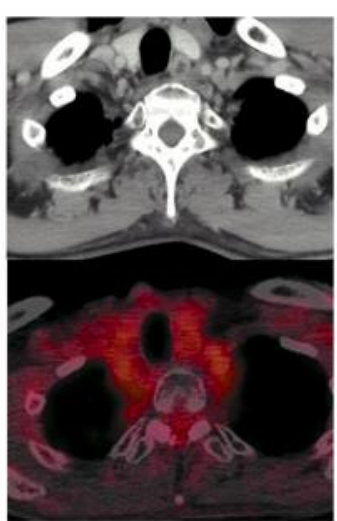

1 Year after CIRT

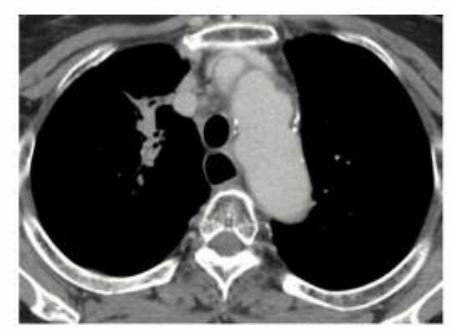

6 Months after CIRT

\section{[ $48.0 \mathrm{~Gy}(\mathrm{RBE}) / 12 \mathrm{fr}$ ]}

Figure 1. A: Computed tomography (CT) scan and positron-emission tomography (PET) imaging of the first case treated before and after treatment. The middle figure shows the dose distribution. The initial target volume was defined as the lymph node recurrence and the nodal region. After a total dose of $36 \mathrm{~Gy}$, the field was limited to only the macroscopic lesions with an added margin of $1.0 \mathrm{~cm}$. B: CT scan and PET imaging of the patient, who was previously irradiated, before and after treatment. The middle figure shows the dose distribution. Local fields with a margin of 1.0 cm around the macroscopic tumor were used.

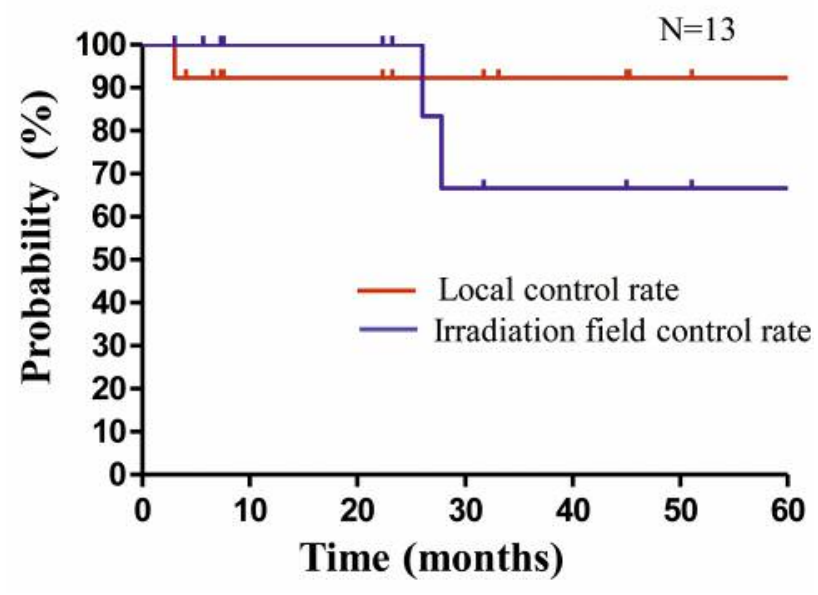

Figure 2. The local control rate and irradiation field control rate curves are illustrated. The 2-, 3-, and 5-year local control rates of these 13 lesions, including salvage re-irradiation, were $92.4 \%$ throughout. The 1-,3-, and 5-year irradiation field control rates were 100.0\%, 66.7\%, and $66.7 \%$, respectively.

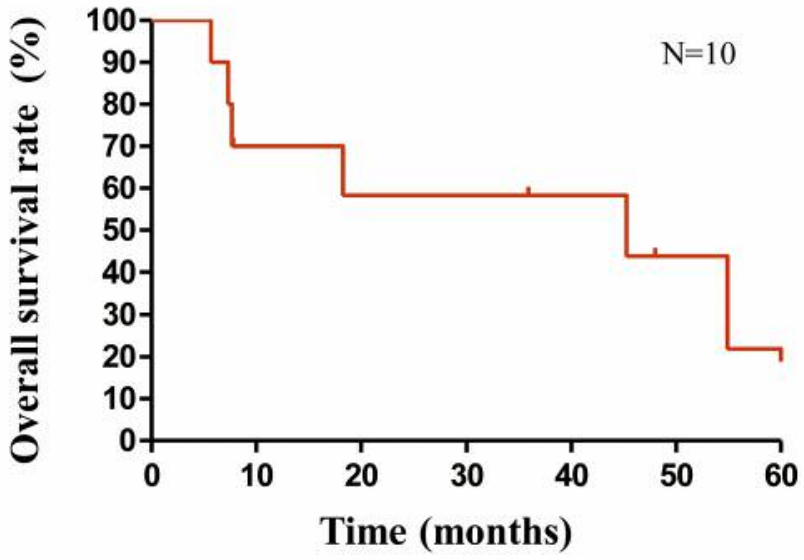

Figure 3. The overall survival rate curves are illustrated. The 1-, 3-, and 5-year survival rates were $70.0 \%, 58.3 \%$, and $21.9 \%$, respectively. Four out of the 10 patients were alive at time of publication. The median survival time was 45.3 months. 
Table II. Clinical outcomes are shown. One patient experienced local recurrence and two patients experienced regional lymph node recurrences within the prophylactic irradiation field. Two patients experienced regional lymph node recurrences out of irradiation field. These two patients received repeat salvage carbon-ion radiotherapy (CIRT).

\begin{tabular}{|c|c|c|c|c|c|c|}
\hline \multirow[b]{2}{*}{ Case } & \multicolumn{6}{|c|}{ Recurrence } \\
\hline & $\begin{array}{l}\text { Radiation } \\
\text { field }\end{array}$ & $\begin{array}{l}\text { Tumor } \\
\text { response }\end{array}$ & Local & $\begin{array}{l}\text { Prophylactic } \\
\text { irradiated field }\end{array}$ & $\begin{array}{c}\text { Outside of } \\
\text { irradiated field }\end{array}$ & $\begin{array}{c}\text { Survival (months), } \\
\text { outcome }\end{array}$ \\
\hline 1 & Local & PR & & & & 7.7 Dead from cancer \\
\hline 2 & Local & PR & & & & 92.1, Dead from pneumonia \\
\hline 3 & Regional & PR & & & $+($ Salvage re-irradiation CIRT) & 54.9, Dead from cancer \\
\hline 4 & Regional & SD & & + & & 45.3, Dead from cancer \\
\hline 5 & Local & PR & & & $+($ Salvage re-irradiation CIRT $\times 2)$ & 48.0, Alive with disease \\
\hline 6 & Regional & CR & & + & & 35.9, Alive with disease \\
\hline 7 & Regional & PD & + & & & 18.3, Dead from cancer \\
\hline 8 & Regional & PR & & & & 7.3, Dead from cancer \\
\hline 9 & Regional & PR & & & & 5.7, Dead from cancer \\
\hline 10 & Local & PR & & & & 7.8, Relapse-free survival \\
\hline
\end{tabular}

CR: Complete response, PR: partial response, SD: stable disease, PD: progressive disease.

example dose distributions with CT images from before and after CIRT in patients 6 and 10 .

Tumor response. The median follow-up duration for all patients and surviving patients was 27.1 (range=5.7-92.1) months and 35.9 (range=35.9-48.0) months, respectively.

Clinical outcomes are shown in Table II. One patient experienced local recurrence and two patients experienced regional lymph node recurrences within the prophylactic irradiation field. Two patients experienced regional lymph node recurrences outside of the irradiation field. These two patients received repeat salvage CIRT. One patient out of the two received salvage CIRT twice, since heterochronic recurrences were detected in para-aortic lymph node and inferior mediastinum after initial CIRT.

Treatment response was complete in one patient, partial in seven, stable one, and with disease progression in one. We analyzed the tumor response including these three salvage lesions. There were eight cases $(62 \%)$ out of 13 cases including salvage cases that achieved complete or partial response after treatment.

The 2-, 3-, and 5-year local control rates of these 13 lesions, including salvage re-irradiation, were $92.4 \%$ throughout. The 1-, 3-, and 5-year irradiation field control rates were $100.0 \%, 66.7 \%$, and $66.7 \%$, respectively. The local control rate and irradiation field control rate curves are illustrated in Figure 2.

The 1-, 3-, and 5-year survival rates were 70.0\%, 58.3\%, and $21.9 \%$, respectively. Four of the 10 patients were alive at time of publication. The median survival time was 45.3 months. The curves for overall survival are illustrated in Figure 3. Five out of the 10 patients $(50.0 \%)$ demonstrated survival of greater than 3 years.
Table III. Acute and late toxicity of the lesions including repeat salvage carbon-ion radiotherapy are listed. No toxicities of grade 2 or more were observed.

\begin{tabular}{lccccccc}
\hline & \multicolumn{3}{c}{ Acute (NCI-CTC) } & & \multicolumn{3}{c}{ Late (RTOG/EORTC) } \\
\cline { 2 - 5 } \cline { 7 - 8 } Toxicity & $\mathrm{n}$ & Grade 0 & Grade 1 & & $\mathrm{n}$ & Grade 0 & Grade 1 \\
\hline Skin & 13 & 6 & 7 & & 13 & 10 & 3 \\
GI tract & 13 & 8 & 5 & & 13 & 13 & 0 \\
Heart & 13 & 13 & 0 & & 13 & 13 & 0 \\
Lung & 13 & 13 & 0 & & 13 & 9 & 4 \\
Spinal cord & 13 & 13 & 0 & & 13 & 13 & 0 \\
Other & 13 & 13 & 0 & & 13 & 13 & 0 \\
\hline
\end{tabular}

NCI-CTC: National Cancer Institute-Common Terminology Criteria, RTOG: Radiation Therapy Oncology Group, EORTC: European Organization for Research and Treatment of Cancer, Gr: grade; GI: gastrointestinal.

Toxicity. All patients completed their CIRT course without interruption. Acute and late toxicity of the 13 lesions including repeat salvage CIRT are listed in Table III. No grade 3 or more acute toxicities were observed.

\section{Discussion}

Our study showed the efficacy and safety of CIRT for patients with isolated lymph node recurrence after curative resection of esophageal cancer. In 10 patients, with a median follow-up of 27.1 months, the 3-year local control rate and overall survival rate were $92.4 \%$ and $58.3 \%$, respectively. CIRT achieved favorable local control and survival, comparable with surgery. Simultaneously, there were no 
grade 3 or higher toxicities reported. This suggests that CIRT is a safe technology providing favorable outcomes in the setting of oligometastatic lymph node recurrence following curative-intent resection of esophageal cancer.

Recent data suggests that chemoradiation (CRT) can improve patient survival in this disease setting $(2,11,21$, 22). Lymphadenectomy tends to be the treatment of choice for isolated lymph node recurrence, and shows good local disease control $(10,11)$. However, challenges remain for achieving favorable long-term outcomes, except in the case of cervical node recurrence $(10,11)$. Nakamura et al. reported a comparison of clinical outcomes with multimodal treatment for recurrent lymph node metastasis, such as lymphadenectomy, conventional CRT and chemotherapy (11). The survival rate at 3 years for groups treated with conventional CRT versus lymphadenectomy were $26.6 \%$ $(\mathrm{n}=21)$ and $50.7 \%(\mathrm{n}=19)$, respectively. Lymphadenectomy tended to offer more favorable survival in comparison with conventional CRT, although no statistically significant difference in survival was noted. The patients who underwent $\mathrm{R} 1$ resection did not survive long term, and it was suggested that CRT might be indicated for lymph node recurrences for which there is a risk of residual disease.

Previous CRT studies for postoperative lymph node recurrence from esophageal cancer demonstrate a median overall survival rate at 3 years of $24 \%$ (range $=11-50.6 \%$ ), with a median survival time of 17 (range=7-43.4) months $(2$, 11, 22-27). Grade 3-5 acute or late toxicities were observed in $0-9 \%$ of patients in these previous studies $(2,11,21-26)$. The clinical outcomes of our study appeared to achieve favorable survival with less toxicity, comparable or superior to other CRT studies.

There were several differing points between our study and previous chemoradiation studies. Firstly, our study included patients who had already received radiotherapy. During the past few decades, preoperative CRT has been actively performed, and a fair number of patients received preoperative radiation $(14,27)$. Generally, re-irradiation is challenging because radiosensitive organs may have already received near-tolerance dose during prior treatments. This makes salvage conventional CRT particularly difficult. CIRT, however, offers a superior dose distribution due to the nature of particulate irradiation, with dose asymptotically concentrated in the Bragg peak (12). This produces a more conformal dose distribution with steeper dose gradients, without an increase in normal tissue integral dose compared with photon. These characteristics enable good local control while diminishing the likelihood of adverse events. Furthermore, two patients with new lymph node recurrence following CIRT were able to receive repeat salvage CIRT with good tolerability and little toxicity.

There are a few limitations to this study. This was a retrospective analysis with a small sample size. Prospective evaluation of a larger cohort will be needed to further verify the results found here.

A number of important issues were defined by our study that will need to be elucidated prior to treatment progression. The control rate within the prophylactic irradiation field at 3 years was limited to $66.7 \%$, with two patients out of 10 developing new regional lymph node recurrences within the prophylactic field. The role of prophylactic irradiation for regional lymph nodes is controversial, and re-evaluation of prophylactic CIRT in this setting may be warranted. To further mitigate locosystemic progression, chemotherapy should be included.

To our knowledge, this is the first study to evaluate the use of CIRT for isolated lymph node recurrence following curative resection of esophageal cancer. Our results were comparable or superior to those for conventional CRT, with no severe toxicities being observed. In particular, CIRT may be a good option for patients who have received prior irradiation to the area of a recurrence. We recommend continued investigation into further CIRT indications and techniques so as to further improve disease response and survival.

\section{Ethical Statement}

The present study was performed at the Hospital of the National Institute of Radiological Science, National Institutes for Quantum and Radiological Science and Technology in accordance with the Declaration of Helsinki and the protocol was approved by the Ethics Committee of the Hospital of the National Institute of Radiological Science, National Institutes for Quantum and Radiological Science and Technology.

\section{Conflicts of Interest}

The Authors declare that they have no conflicts of interest in regard to this study.

\section{Acknowledgements}

This study was supported by the Working Group for Esophageal Cancer at the National Institute of Radiological Science consisted of multiple facilities: Chiba University (Hisahiro Matsubara, Chairman, Yasunori Akutsu, Kentaro Murakami, Takashi Uno, Yukio Nakatani), NIRS (Hirohiko Tsujii, Tadashi Kamata, Hiroshi Tsuji, Shigeru Yamada, Shigeo Yasuda), Chiba Cancer Center (Matsuo Nagata, Yoshihiro Nabeya, Isamu Hoshino), Toho University (Hideaki Shimada Shinichi Okazum), Toranomon Hospital (Harushi Udagawa), Yamagata University (Kenji Nemoto), Tokyo University (Yasuyuki Seto), Tokyo Medical and Dental University (Tatsuyuki Kawano), Keio University (Yuko Kitagawa), Kanagawa Cancer Center (Takashi Ogata), Cancer Institute Hospital (Masayuki Watanabe), Gunma University (Hiroyuki Kuwano).

\section{References}

1 Siegel RL, Miller KD and Jemal A: Cancer statistics, 2016. CA Cancer J Clin 66(1): 7-30, 2016. 
2 Jingu K, Ariga H, Nemoto K, Narazaki K, Umezawa R, Takeda K, Koto M, Sugawara T, Kubozono M, Miyata G, Onodera K and Yamada S: Long-term bresults of radiochemotherapy for solitary lymph node metastasis after curative resection of esophageal cancer: Inter Jour Radia Oncol 83(1): 172-177, 2012.

3 Nakagawa S, Kanda T, Kosugi S, Ohashi M, Suzuki T and Hatakeyama K: Recurrence pattern of squamous cell carcinoma of the thoracic esophagus after extended radical esophagectomy with three-field lymphadenectomy. J Am Coll Surg 198: 205211, 2004

4 Law SY, Fok M, Wong J: Pattern of recurrence after oesophageal resection for cancer: clinical implications. Br J Surg 83: 107 $111,1996$.

5 Bhansali MS, Fujita H, Kakegawa T, Yamana H, Ono T, Hikita S, Toh Y, Fujii T, Tou U and Shirouzu K: Pattern of recurrence after extended radical esophagectomy with three-field lymph node dissection for squamous cell carcinoma in the thoracic esophagus. World J Surg 21: 275-281, 1997.

6 Kato H, Miyazaki T, Nakajima M, Sohda M, Fukai Y, Masuda N, Fukuchi M, Manda R, Tsukada K and Kuwano H: Prediction of hematogenous recurrence in patients with esophageal carcinoma. Jpn J Thorac Cardiovasc Surg 51: 599-608, 2003.

7 Hulscher JB, van Sandick JW, Tijssen JG, Obertop H and van Lanschot JJ: The recurrence pattern of esophageal carcinoma after transhiatal resection. J Am Coll Surg 191: 143-148, 2000.

8 Kyriazanos ID, Tachibana M, Shibakita M, Yoshimura H, Kinugasa S, Dhar DK, Nakamoto T, Fujii T and Nagasue N: Pattern of recurrence after extended esophagectomy for squamous cell carcinoma of the esophagus. Hepatogastroenterology 50: 115$120,2000$.

9 Natsugoe S, Matsumoto M, Okumura H, Uchikado Y, Setoyama T, Sasaki K, Sakurai T, Omoto I, Owaki T, Shinchi H, Ueno S and Ishigami $S$ : Clinical course and outcome after esophagectomy with three-field lymphadenectomy in esophageal cancer. Langenbecks Arch Surg 395(4): 341-346, 2010.

10 Watanabe M, Mine S, Yamada K, Shigaki H, Baba Y, Yoshida N, Kajiyama K, Yamamoto N, Sano T and Baba H: Outcomes of lymphadenectomy for lymph node recurrence after esophagectomy or definitive chemoradiotherapy for squamous cell carcinoma of the esophagus. Gen Thorac Cardiovasc Surg 11: 685-692, 2014.

11 Nakamura T, Ota M, Narumiya K, Sato T, Ohki T, Yamamoto M and Mitsuhashi N: Multimodal treatment for lymph node recurrence of esophageal carcinoma after curative resection. Ann Surg Oncol 15(9): 2451-2457, 2008.

12 Tsujii H, Kamada T: A review of update clinical results of carbon ion radiotherapy. Jpn J Clin Oncol 42(8): 670-685, 2012.

13 Hall EJ and Giaccia AJ: Radiobiology for the Radiologist. Philadelphia, PA: Lippincott Williams \& Wilkins, 2012.

14 Akutsu Y, Yasuda S, Nagata M, Izumi Y, Okazumi S, Shimada H, Nakatani Y, Tsujii H, Kamada T, Yamada S and Matsubara $\mathrm{H}$ : A phase I/II clinical trial of preoperative short-course carbonion radiotherapy for patients with squamous cell carcinoma of the esophagus. J Surg Oncol 105(8): 750-755, 2012.

15 Tsujii H, Kamada T, Shirai T, Noda K, Tsuji H and Karasawa K: Carbon-Ion Radiotherapy, Springer Science \& Business Media, Tokyo, 2013.

16 Kanda Y: Investigation of the freely available easy-to-use software 'EZR' for medical statistics. Bone Marrow Transplant 48(3): 452-458, 2013.
17 Inaniwa T, Kanematsu N, Matsufuji N, Kanai T, Shirai T, Noda K, Tsuji H, Kamada T and Tsujii H: Reformulation of a clinicaldose system for carbon-ion radiotherapy treatment planning at the National Institute of Radiological Sciences, Japan. Phys Med Biol 60(8): 3271-3286, 2015.

18 Eisenhauer E. A, Therasse P, Bogaerts J, Schwartz LH, Sargent D, Ford R, Dancey J and Arbuck S: New Response Evaluation Criteria in Solid Tumours: Revised RECIST guideline (version 1.1). Eur J Cancer 45: 228-247, 2009.

19 NCI Common Terminology Criteria for Adverse Events (CTCAE) v4.0 data files. Available from URL: http:// evs.nci.nih.gov/ftp1/CTCAE/About.html, 2017.

20. Cox JD, Stetz J and Pajak TF: Toxicity criteria of the Radiation Therapy Oncology Group (RTOG) and the European Organization for Research and Treatment of Cancer (EORTC). Int J Radiat Oncol Biol Phys 31: 1341-1346, 1995.

21 Jingu K, Matsushita H, Takeda K, Umezawa R, Takahashi C, Sugawara T, Kubozono M, Abe K, Tanabe T, Shirata Y, Yamamoto T, Ishikawa Y and Nemoto K: Long-term results of radiotherapy combined with nedaplatin and 5-FU for postoperative loco-regional recurrent esophageal cancer: Update on phase II study. BMC Cancer 12: 542, 2012.

22 Yamashita H, Jingu K, Niibe Y, Katsui K, Matsumoto T, Nishina $\mathrm{T}$ and Terahara A: Definitive salvage radiation therapy and chemoradiation therapy for lymph node oligo-recurrence of esophageal cancer: a Japanese multi-institutional study of 237 patients. Radiat Oncol 12: 38, 2017.

23 Nemoto K, Ariga H, Kakuto Y, Matsushita H, Takeda K, Takahashi C, Takai Y, Yamada S and Hosoi Y: Radiation therapy for locoregionally recurrent esophageal cancer after surgery. Radiother Oncol 61: 165-168, 2001.

24 Yamashita H, Nakagawa K, Tago M, Nakamura N, Shiraishi K and Ohtomo K: Salvage radiotherapy for postoperative locoregional recurrence of esophageal cancer. Dis Esophagus 18: 215-220, 2005.

$25 \mathrm{Lu}$ JC, Kong C and Tao H: Radiotherapy with or without concurrent chemotherapy for lymph node recurrence after radical surgery of thoracic esophageal squamous cell carcinoma. Int J Radiat Oncol Biol Phys 78: 710-714, 2010.

26 Maruyama K, Motoyama S, Anbai A, Usami S, Sato Y, Shibuya $\mathrm{K}$, Yoshino $\mathrm{K}$, Nakatsu $\mathrm{T}$, Minamiya $\mathrm{Y}$ and Ogawa JI: Therapeutic strategy for the treatment of postoperative recurrence of esophageal squamous cell carcinoma. Dis Esophagus 15: 2451-2547, 2011.

27 van Heijl M, van Lanschot JJ, Koppert LB, van Berge Henegouwen MI, Muller K, Steyerberg EW, van Dekken H, Wijnhoven BP, Tilanus HW, Richel DJ, Busch OR, Bartelsman JF, Koning CC, Offerhaus GJ and van der Gaast A: Neoadjuvant chemoradiation followed by surgery versus surgery alone for patients with adenocarcinoma or squamous cell carcinoma of the esophagus (CROSS). BMC Surg 8: 21, 2008. 